\title{
Narrativa
}

Nuova serie

39 | 2017

Nuove frontiere del Sud

\section{Tre Sud di Ernesto de Martino}

\section{Roberto Dainotto}

\section{(2) OpenEdition}

\section{Journals}

\section{Edizione digitale}

URL: https://journals.openedition.org/narrativa/660

DOI: $10.4000 /$ narrativa.660

ISSN: 2804-1224

\section{Editore}

Presses universitaires de Paris Nanterre

\section{Edizione cartacea}

Data di pubblicazione: 1 décembre 2017

Paginazione: 53-64

ISBN: 978-2-84016-289-6

ISSN: 1166-3243

Notizia bibliografica digitale

Roberto Dainotto, «Tre Sud di Ernesto de Martino», Narrativa [Online], 39 | 2017, online dal 01

décembre 2021, consultato il 14 janvier 2022. URL: http://journals.openedition.org/narrativa/660 ; DOI: https://doi.org/10.4000/narrativa.660

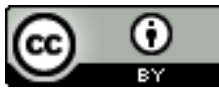

Narrativa est mise à disposition selon les termes de la Licence Creative Commons Attribution 4.0 International. 


\section{Tre Sud di Ernesto de Martino}

... si è profilata una minaccia relativistica che impone di ristabilire l'universalmente umano che opera nella varietà delle situazioni esistenziali. È certamente vero che l'uomo teorizzato dalle forme tradizionali di vita culturale era per esempio soltanto il maschio, l'adulto, l'europeo, il colonizzatore, il sano, il rappresentante di strati sociali egemoni: ma la polemica contro queste limitazioni del vecchio umanesimo non deve significare la perdita di quel senso fondamentale che sempre di nuovo ristabilisce una comune misura dell'umano ${ }^{1}$.

1. L'attenzione con cui Ernesto de Martino seguiva la pubblicazione delle opere di Antonio Gramsci dal $1948^{2}$ era maturata nella scelta di un preciso campo di studi di chiara ascendenza gramsciana (la questione meridionale intesa come "comprensione delle condizioni materiali di esistenza dei contadini del Sud d'Italia"3) e di un altrettanto gramsciana problematica: come può l'intellettuale, espressione di una cultura egemone, "studiare [...] gli elementi della psicologia popolare"'?

La problematica era spesso, come Gramsci sapeva, pretesto di false soluzioni: o "per una élite sociale, gli elementi dei gruppi subalterni hanno... alcunché di barbarico e di patologico"s; oppure l'intellettuale concede loro dignità di culture specifiche nella loro unicità, rischiando però "una forma di relativismo"'.

1. De Martino, Ernesto, La fine del mondo. Contributo all'analisi delle apocalissi culturali, Torino, Einaudi, 2002, p. 265.

2. Cfr. Pavanello, Mariano, "Il tarantismo osservato. Ricerca sul terreno e teoria in Ernesto de Martino”, in La ricerca folklorica, n. 67/68, 2013, p. 31.

3. Pizza, Giovanni, "Gramsci e de Martino. Appunti per una riflessione”, in Quaderni di teoria sociale, 13, 2013, p. 79.

4. Gramsci, Antonio, Quaderni del carcere, Torino, Einaudi, 1975, Q3\$48.

5. Ibid., Q25\$1.

6. Ibid., Q8\$156. 
Se così il positivismo affermava essere "i meridionali [...] biologicamente degli esseri inferiori, dei semibarbari o dei barbari completi" ${ }^{\prime}$, i folcloristi, mutatis mutandis, li studiava come "una bizzarria, una stranezza". Ma l'aut aut non risolveva la questione. Se nessuna cultura può presumere "ancora di essere la premessa necessaria, universale di ogni modo di pensare e di agire", questo non significa dovere universalizzare il relativismo stesso. "L'argomento del pericolo del relativismo [...] non è valido"": non si tratta di risolvere la problematica für ewig, bensì di operare in una tensione storica, dialettica, tra intellettuali e popolo; in quella dialettica, cioè a dire, che Gramsci amava chiamare, in polemica con gli studiosi del mondo popolare, "con-sentire". Suo fine: "una catarsi di civiltà moderna" 10 che segnasse il momento dell'unità avvenuta tra intellettuale e popolo, tra osservatore e osservato.

A ben vedere, e con ripercussioni notevoli sul pensiero di de Martino, fine della catarsi era anche un altro: riunificare cioè le ambizioni dei gruppi subalterni, la cui storia "è necessariamente disgregata ed episodica" ${ }^{11}$, in forza comune e quindi egemone ${ }^{12}$. Se così la subalternità contadina del meridione domandava il "con-senso" dell'avanguardia intellettuale torinese, al contempo quello stesso "Meridione [...] mercato di vendita semicoloniale" ${ }^{13}$ andava ripensato anche in relazione ad altri contesti "coloniali e semicoloniali: India, negri dell'Africa, ecc." ${ }^{14}$.

La problematica gramsciana, insomma, consisteva nella scommessa di organizzare subalternità disperse in nuovo "soggetto politico" 15 : riformare la loro "grande disgregazione sociale"16 in nuova e comune "soggettività [...] oggettivata e universalizzata concretamente" ${ }^{17}$. Nelle parole di de Martino, si trattava di creare "di nuovo", pure contro il "maschio, l'adulto, l'europeo, il colonizzatore,

7. Gramsci, Antonio, Nel mondo grande e terribile, Torino, Einaudi, 2007, p. 118.

8. ID., Quaderni del carcere, cit., Q1ฐ89.

9. Ibid., Q8\$156.

10. Ibid., Q4\$33.

11. Ibid., Q25\$1.

12. "ogni atto storico [...] presuppone il raggiungimento di una unità 'culturalesociale' per cui una molteplicità di voleri disgregati, con eterogeneità di fini, si saldano insieme per uno stesso fine" (Ibid., Q10II\$44).

13. Ibid., Q1ฐ43.

14. Ibid., Q2ऽ47.

15. Burgio, Alberto, Gramsci. Il Sistema in Movimento, Roma, DeriveApprodi, 2014, p. 352.

16. Gramsci, Antonio, Nel Mondo grande e terribile, cit., p. 137.

17. ID., Quaderni del carcere, cit., Q8\$177. 
il sano, il rappresentante di strati sociali egemoni”, le premesse di "una comune misura dell'umano" 18 .

Nel fare della problematica di Gramsci "il problema mio"19, de Martino ambiva così a situare il "paradosso dell'incontro etnografico" 20 in un terreno che non fosse né quello dell'etnocentrismo europeo, né quello di un relativismo assoluto, bensì quello di una dialettica. Questa si svolgeva, in prima battuta, in incontro tra cultura egemone e cultura subalterna:

l'incontro etnografico che acquista particolare rilievo nell'Italia di oggi è quello con il mondo contadino del Sud, sotto la spinta dell'attuale fase della quistione meridionale. Non si tratta di isolare tale mondo dal resto della società meridionale e dalla storia culturale del nostro paese, e ancor meno di isolare quanto di più arcaico e tradizionale permane in esso, per poi restare prigionieri di questi prodotti dell'astrazione: al contrario, la particolare dialettica racchiusa nell'incontro etnografico comporta, qui come altrove, una duplice misurazione, che si rivolge sia all'ethnos e al suo condizionamento culturale, sia alla civiltà occidentale rispetto alla quale l'ethnos sta come documento mediatore di nuove vie di consapevolezza storica ${ }^{21}$.

A una dialettica tra "civiltà occidentale" e mondo "arcaico", ne seguiva quindi un'altra: tra Meridione italiano e i Sud del mondo "vanno sempre più attenuandosi le ragioni che $[\ldots]$ avevano consigliato di assegnare a discipline diverse $\mathrm{i}$ popoli 'primitivi' e le plebi rustiche europee"22.

Questa esigenza di individuare "raccordi, passaggi, sincretismi" 23 tra Mezzogiorno e altri Sud nasceva in de Martino in un momento storico forse determinante: nell'Italia della Ricostruzione, ancora irrisolto era "quel complesso di problemi sociali, politici e culturali che va sotto il nome di questione

18. de Martino, Ernesto, La fine del mondo, cit., p. 265.

19. "Dopo il mio incontro con gli uomini della Rabata, ho riflettuto che non c'era soltanto un problema loro, il problema della loro emancipazione, ma c'era anche il problema mio, il problema dell'intellettuale piccolo-borghese del Mezzogiorno, con una certa tradizione culturale e una certa 'civiltà' assorbita nella scuola, e che si incontrava con questi uomini ed era costretto per ciò stesso ad un esame di coscienza, a diventare per così dire l'etnologo di se" (ID., Furore simbolo valore, Milano, Feltrinelli, 2002, p. 132).

20. ID., La fine del mondo, cit., p. 391.

21. ID., Furore simbolo valore, cit., p. 90.

22. Ibid., pp. 73-74.

23. Id., Sud e magia, Milano, Feltrinelli, 2001, p. 8. 
meridionale"24; nel contesto internazionale emergevano invece, "scandalo" per l'Europa facente fronte alla "sfida di tale alienità" 25 , i processi di decolonizzazione. Nell'estate del 1948, recensendo Miti e leggende di Raffaele Pettazzoni, de Martino coglieva più che una affinità tra l'antica problematica del Meridione "Africa italiana" ${ }^{26}$ e le ex-colonie. Ma se queste, frutto di una guadagnata "coscienza di essere prigionieri di un umanesimo circoscritto", segnavano adesso "l'irruzione di fatto nella storia dei popoli coloniali", bisognava ancora portare in Italia, con "Levi da una parte e Gramsci dall'altra" 27, le "esperienze del mondo che (per riprendere l'immagine che piacque al Levi) 'vive oltre Eboli"”28. Insomma, "il modo di essere 'etnologico' delle plebi meridionali, che le rendeva affini, nella concretezza della loro realtà sociale ed umana, a tutte le altre plebi del 'terzo mondo', conteneva [...] un ammonimento che aveva anche un significato politico, che aveva anzi soprattutto un significato politico" 29 .

Per questa ragione, e per il mutato contesto rispetto ai Quaderni, una "traduzione" ${ }^{30}$ dei processi decoloniali nel contesto nazionale si faceva per de Martino tanto più urgente che in Gramsci. Così urgente da richiedere, in una glossa del 1949, emendamenti a Gramsci stesso:

occorre aggiungere dell'altro e correggere e chiarificare in più parti il punto di vista gramsciano.

È necessario unificare idealmente la storia delle classi subalterne per entro le nazioni che appartengono alla civiltà euro-americana, e la storia delle civiltà coloniali e semicoloniali con le quali la civiltà euro-americana si è incontrata nel corso del suo sviluppo capitalistico ${ }^{31}$.

24. ID., Morte e pianto rituale nel mondo antico. Dal lamento funebre pagano al pianto di Maria, Torino, Bollati Boringhieri, 2008, p. 319.

25. ID., La fine del mondo, cit., p. 395.

26. Cfr. Petraccone, Claudia, Le due civiltà. Settentrionali e meridionali nella storia d'Italia dal 1860 al 1914, Bari, Laterza, 2000, pp. 6-12.

27. SATTA, Gino, "Etnografia e politica", in nostos, n. 1, dicembre 2016, p. 384.

28. de Martino, Ernesto, "Recensione a Miti e leggende", in Avanti!, LII, 157, 4 luglio 1948, p. 3.

29. Galasso, Giuseppe, Croce, Gramsci e altri storici, Milano, Il Saggiatore, 1978, p. 383.

30. Cfr. Frosini, Fabio, "Sulla 'traducibilità' nei Quaderni di Gramsci”, in Critica marxista, NS, n. 6, 2003.

31. De Martino, Ernesto, “Due inediti su Gramsci: 'Postille a Gramsci’ e 'Gramsci e il Folklore"”, in La ricerca folkelorica, n. 25, aprile 1992, p. 74. 
Bisognava insomma non solo intendere come "mondo popolare subalterno" l'insieme "dei popoli coloniali e semicoloniali, e del proletariato operaio e contadino delle nazioni egemoniche", ma soprattutto, per quanto riguarda la subalternità meridionale, "unificare di fatto la sua storia con la storia" 32 .

2. Per queste ragioni, "Sud" (o "Meridione") non doveva assumere il valore di una designazione relativa, bensì essere reso partecipe a la storia dei popoli decolonizzati: 'il termine 'sud' non ritiene il valore di una designazione meramente geografica, ma politica e sociale" ${ }^{\prime 3}$. Diveniva cioè concetto capace di contenere in sé le realtà coloniali e semicoloniali; funzionale a ripensare e riorganizzare "molecolarmente" ${ }^{44}$ diversità plurime, ma tutte sotto il giogo dello stesso capitalismo che le sussumeva nelle sue diverse forme, in soggettività politica. Cioè: in "coscienza".

Proprio sulla questione della coscienza si sarebbe prodotta un'incrinatura nei rapporti tra de Martino e il maestro Benedetto Croce. Distinguendo Kultur- da Naturvölker, e negando ai secondi ogni capacità di autocoscienza e produzione culturale, Croce mostrava

in tutta la sua spietata crudezza, la ideologia borghese verso i popoli coloniali, con i quali certamente non ci può essere, in questo rapporto pratico, di dominio, comunanza di memorie e comprensione storica effettiva. L'etnos appare qui come una folla di "anime morte", ma in realtà a renderle "morte" ha provveduto la prassi della conquista e del dominio, il rapporto naturalistico che l'imperialismo coloniale instaura necessariamente fra "civiltà" e "barbarie" 35.

E non era solo la coscienza culturale dei popoli coloniali che premeva qui segnalare: "non sostanzialmente diverso fu l'incontro fra lo Stato italiano e l'etnos del Mezzogiorno e delle isole"

Qui sorgeva il problema: se "quel movimento di emancipazione dal colonialismo $[\ldots]$ costituisce uno dei più importanti eventi dell'epoca moderna" ${ }^{\prime 3}$,

32. Ibid., p. 411.

33. ID., Sud e magia, cit., p. 8.

34. Forenza, Eleonora, "Molecolare", in Liguori, Guido, Voza, Pasquale (a cura di), Dizionario gramsciano 1926-1937, Roma, Carocci, 2009.

35. De Martino, Ernesto, "Etnologia e cultura nazionale negli ultimi dieci anni", in Società, IX, n. 3, settembre 1953, p. 317.

36. Ibid., p. 318.

37. ID., "Nota introduttiva" a LANTERnARI, Vittorio, La grande festa, Milano, il Saggiatore, 1959, p. 16. 
bisognava ancora capire perché nel Mezzogiorno non si produceva ancora una "coscienza di essere prigionieri di un umanesimo circoscritto"38; "coscienza della... situazione reale e delle contraddizioni che la caratterizzano" 39 . È plausibile ipotizzare dunque che è a partire da questo nodo che nasca in de Martino l'esigenza di legare i concetti di coscienza, subalternità e Sud alla categoria ${ }^{40}$ di crisi: "crisi della presenza" e "crisi della personalità" caratterizzano precisamente la mancanza di coscienza dell'esperienza subalterna e, allo stesso tempo, aprono la possibilità di una risoluzione di questa in una presa di coscienza.

Di “crisi della presenza" parla il Mondo magico del 1948 per concettualizzare la condizione esistenziale di una umanità caduta nell"“ingens sylva della natura" la "presenza" (traduzione di Dasein nell'edizione italiana di Sein und Zeit di Martin Heidegger), l'esserci dell'essere umano nel mondo, è costantemente minacciata dagli imprevisti, dall'indifferenza, dal caos stesso della vita naturale. La crisi pone quindi a individui e comunità l'imperativo di una sua risoluzione: un "bisogno di senso" $" 42$ che riduca a forme culturali intellegibili (il rito, il magico, ecc.) la natura ostile, che così diventa "'natura culturalmente condizionata', cioè [...] valutabile come istituto, sorretto da intenzioni umane" 43 .

Se è vero che la categoria di crisi allude qui al dramma heideggeriano della "angoscia e la decisione alla morte" ${ }^{44}$, bisogna pure rilevare come essa non coincida con il mero dramma esistenzialista. Non è solo che l'unico riferimento a Sein und Zeit è qui "citazione non proprio benevola" ${ }^{4}$. Il problema sta ben più a monte:

Il comune presupposto di tutti gli autori esistenzialisti è [...] "l'uomo chiuso in un orizzonte inesplicabilmente finito, nel cuore di una esistenza mondana umiliata". Tale limitazione della presenza appare come un dato, oscuro e angoscioso nella sua origine, come un qui e un ora che non dipende da $\mathrm{me}^{46}$.

38. ID., "Recensione a Miti e leggende", cit., p. 3.

39. ID., "Intorno a una storia del mondo popolare subalterno", in Società 5, n. 3 , 1949, p. 419.

40. "sebbene tutte le categorie siano pensabili e pensate come concetti, per sé non sono concetti, ma potenze [dei concetti]" (СROCE, Benedetto, "Osservazioni intorno alla dottrina delle categorie", in Quaderni della Critica, 17/18, 1950, p. 15).

41. De Martino, Ernesto, Il mondo magico. Prolegomeni a una storia del magismo, Torino, Bollati Boringhieri, 2015, p. 173.

42. Battistrada, Franco, Per un umanesimo rivisitato. Da Scheler a Heidegger, da Gramsci a Jonas, all'etica di liberazione, Milano, Jaca Book, 1999, p. 215.

43. De Martino, Ernesto, Il mondo magico, cit., pp. 52-53.

44. CASES, Cesare, "Introduzione" a De MARTINo, Ernesto, Il mondo magico, cit., p. xxviii.

45. PACI, Enzo, "Il nulla e il problema dell'uomo", in DE Martino, Ernesto, Il mondo magico, cit., p. 260.

46. De Martino, Ernesto, Il mondo magico, cit., p. 160. 
Insomma, il problema con Heidegger e con l'esistenzialismo non è tanto che "nessuna nota dell'archivio risulta [...] sufficiente per provare l'esistenza di una lettura diretta del testo heideggeriano" "47, quanto la chiusura dell'orizzonte umano in una "origine" mondana che "appare come un dato". Il problema è l'isolare il dramma umano da quella storia che, vichianamente, è fatta dagli uomini, e che quindi gli uomini possono cambiare. L'esistenzialismo, per ridurre a sentenza, non ha una prassi ${ }^{48}$.

La crisi e il suo riscatto, già nel Mondo magico, si presentano allora non (solo) come esperienza esistenziale, ma come un dramma che "vede il suo giorno nella storia umana"49; e che quindi, presumibilmente, ancora nella storia avrà avuto la sua notte: a minacciare la presenza, non è solo la natura ostile, ma la stessa storia, le stesse istituzioni, le stesse forme fatte dagli uomini che possono ritornare adesso come eventi minacciosi. Non solo carestie, insomma, ma guerre, sfruttamento e dominazione. È per questo che le collettività sentono il bisogno, oltre che di condizionare culturalmente la natura, anche di fuggire dalla storia, di "destorificare" ${ }^{50}$ un minaccioso evento. Sospendendo la storia, il rituale, l'intervento magico, il sacrificio, muovono la comunità in una meta-storia, in un tempo che ritorna come esorcismo del dato ${ }^{51}$.

Se si è insistito su una storicità del dramma umano, è perché sarà proprio nella storia che de Martino potrà sviluppare la categoria di crisi come fondante, non tanto di un'astratta esistenza umana, ma dell'esperienza storica di popoli subalterni, coloniale e semicoloniali. Già nelle "Note di viaggio", che segnano forse il passaggio a una etnologia più marcatamente post-coloniale, crisi sarà l'“insorgere dei momenti critici dell'esistenza storica" ${ }^{52}$; di un'esistenza cioè comune alla storia dei contadini della Lucania come alle esperienze degli "Aranda australiani, degli Yamana e dei Selk'nam della Terra del Fuoco, dei Tungusi della Siberia". Crisi che si trasforma dalle sue origini esistenzialiste in categoria funzionale

47. PÀstina, Roberto, "Le note sull'esistenzialismo", in GALLInI, Clara (a cura di), Ernesto De Martino e la formazione del suo pensiero, Napoli, Liguori, 2005, p. 186.

48. Cfr. Lanternari, Vittorio, "Ernesto De Martino fra storicismo e ontologismo", in Studi storici, 19, n. 1, 1978.

49. de Martino, Ernesto, Il mondo magico, cit., p. 74.

50. Ibid., p. 233.

51. Cfr. Ferrari, Fabrizio, Ernesto De Martino on Religion: The Crisis and the Presence, Bristol, Equinox, 2012, p. 18.

52. Berrocal, Emilio, "The Post-Colonialism of Ernesto De Martino: The Principle of Critical Ethnocentrism as a Failed Attempt to Reconstruct Ethnographic Authority", in History and Anthropology, vol. 20, n. 2, giugno 2009, p. 124. 
a concettualizzare "un concreto mondo culturale di oppressi (come che sia determinata questa oppressione, dalla natura o dagli uomini)" ${ }^{53}$.

3. D'altra parte, come de Martino aveva messo in chiaro nel polemico "Crisi della presenza e reintegrazione religiosa", le sue categorie, a differenza di quelle crociane, non volevano essere trascendentali ma storiche ${ }^{54}$. Diventa allora plausibile considerare, in un pensatore di cui è riconosciuta la "costante capacità di riplasmare le suggestioni più diverse" ${ }^{55}$, che la categoria di crisi posta nel Mondo magico si vada complicando di plurime suggestioni e in risposta a contesti sempre nuovi.

Tra Mondo magico (1948) e Sud e magia (1959) intercorrono eventi storici di notevole portata (recedere delle lotte agrarie, riforme socialdemocratiche, guerra fredda, miracolo economico, rivoluzioni coloniali), come anche una serie di letture nell'ambito del marxismo (Gramsci) che complicano la categoria di crisi. Questa è adesso sintomo della seguente lista:

la precarietà dei beni elementari della vita, l'incertezza delle prospettive concernenti il futuro, la pressione esercitata sugli individui da parte di forze naturali e sociali non controllabili, la carenza di forme di assistenza sociale, l'asprezza della fatica nel quadro di una economia agricola arretrata, l'angusta memoria di comportamenti razionali efficaci con cui fronteggiare realisticamente i momenti critici dell'esistenza ${ }^{56}$.

Pochi anni più tardi, nello studio del tarantismo salentino (negli anni del "miracolo" e in quelli della Dicbiarazione sulla decolonizzazione delle Nazioni Unite), la categoria di crisi si colora di nuove sfumature ancora.

Se la questione del colonialismo era caduta in secondo piano in Sud e magia, ritorna adesso nell'analisi del meridione:

Quando, dal 1561 in poi, i gesuiti intrapresero la loro attività missionaria nel vicereame di Napoli [...] venne spontaneamente alle loro labbra l'espressione "India italiana" per designare questa parte d'Italia [...] Questa coscienza culturale

53. De Martino, Ernesto, "Note di viaggio", in Nuovi Argomenti, vol. I, n. 2, 1953, p. 47.

54. Cfr. Berardini, Sergio, "de Martino, Croce, e il problema delle categorie", in Pozzoni, Ivan (a cura di), Benedetto, Croce. Teoria e orizzonti, Villasanta, Limina Mentis, 2013.

55. Cherchi, Placido, Cherchi, Maria, Ernesto De Martino: dalla crisi della presenza alla comunità umana, Napoli, Liguori, 1987, p. 252.

56. de Martino, Ernesto, Sud e magia, cit. p. 89. 
gesuitica dell'Italia meridionale come Indias de por acá costituisce qualche cosa di più di una metafora occasionale, poiché segnala una effettiva analogia, nella prospettiva dell'attività missionaria, fra il nuovo mondo da guadagnare alla civiltà cristiana e le condizioni culturali di larghe zone del vicereame di Napoli, così superficialmente sfiorate da questa civiltà che i suoi abitanti sembravano "tutti del bosco" 57 .

È un'analoga crisi storica, nelle colonie come nelle semicolonie, a produrre la crisi. Non si tratta solo di un Io schiacciato dalla natura e non più padrone del proprio destino (quindi in preda alla delusione di essere posseduto da demoni o tarantole); né semplicemente della crisi storica di un'economia arretrata. Nella semicolonia dell'ex regno di Napoli come nelle colonie ultramarine, la crisi riguarda comunità intere i cui riferimenti culturali sono posseduti essi stessi dalla cultura egemone (il cristianesimo o la scienza medica nel Salento), e le cui culture autoctone vengono liquidate a "relitto" 58 , a non-cultura dei boschi. La "profonda disgregazione" del mondo subalterno, la crisi, rischia così di essere totale: individuale, sociale, economica, politica, e culturale. Ciò che resta è il tarantismo nel Salento come, altrove, "culti africani strutturalmente affini (zar, bori etc.) caratterizzati dalla possessione da parte di dèmoni" e che anche nel "mondo afroamericano [...] si sono sviluppati con modalità particolari e assumendo vari nomi (macumba, candomblé, santería, vodu)" 59 .

Nel frattempo, altro si è aggiunto anche alle letture demartiniane: L'Afrique fantôme di Michel Leiris, Afrique ambigue di Georges Balandier, e Le Vaudou haïtien di Alfred Métraux in particolare che, oltre a discutere fenomeni analoghi di possessione, sembrano quasi voler raccordare la categoria demartiniana di crisi all'engagement anti-colonialista che in quegli anni andava costituendo lo spazio privilegiato della ricerca di Les Temps modernes:

l'espace exemplaire de l'engagement forme, dans Les Temps Modernes, une géographie très particulière qui souligne, valorise et relie en quelque façon ce que l'on pourrait nommer "les trois Suds". D'abord l'Afrique, avec Michel Leiris, Georges Balandier et, surtout, le psychiatre noir martiniquais Frantz Fanon [...]. Puis son prolongement américain: soit les Antilles de Leiris et Métraux, bien sûr, et le Sud des États-Unis qui est, avec ses écrivains, William Faulkner et Richard Wright, mais aussi James Agee (dont Les Temps Modernes

57. ID., La terra del rimorso. Contributo a una storia religiosa del Sud, Milano, Saggiatore, 2002, pp. 43-44.

58. Ibid., p. 46.

59. Ibid., pp. 206-207. 
feront connaître [...] l'ouvrage signé avec Walker Evans [...]. Enfin le Sud italien qui prendra vite dans le triptyque une place équivalente. En décembre 1946, un chapitre de Cristo si è fermato a Eboli de Carlo Levi est publié dans la revue ${ }^{60}$.

Qui sorge però una perplessità: perché, assente nella Terra del rimorso, rimane proprio il nome di Frantz Fanon ${ }^{61}$ ? Sulle pagine di Temps modernes, Fanon aveva pubblicato Peaux noires, masques blancs. Così vi descriveva il tipo nevrotico del colonizzato:

Le nègre dans son comportement s'apparente à un type névrotique obsessionnel ou, si l'on préfère, il se place en pleine névrose situationnelle. Il y a chez l'homme de couleur tentative de fuir son individualité, de néantiser son être-làa ${ }^{62}$.

Si trattava insomma di quella nevrosi che diventerà il tema di "Guerre coloniale et troubles mentaux":

Parce qu'il est une négation systématisée de l'autre, une décision forcenée de refuser à l'autre tout attribut d'humanité, le colonialisme accule le peuple dominé à se poser constamment la question: "Qui suis-je en réalité?"33.

Non era questo annientamento dell'io colonizzato precisamente "raccordabile" alla crisi esperita dal contadino meridionale di fronte alla natura nemica (che nega un buon raccolto) e di fronte l'inumana storia (del capitalismo agrario)? Non era l'annientamento del soggetto nègre lo stesso del bracciante del Salento, che somatizzava nella nevrosi, come scriveva lo psichiatra della Terra del rimorso, "l'impossibilità di divenire soggetto politico" "64?

Fanon, del resto, era presenza non inavvertibile nell'Italia del miracolo. Il suo pensiero circolava qui più speditamente e liberamente che in Francia ${ }^{65}$, dacché, nel dopoguerra democristiano, la rivoluzione algerina veniva spesso associata per simpatia alla Resistenza (tradita: ecco il rimorso del meridione). Nel 1959

60. FABRE, Daniel, "Un rendez-vous manqué. Ernesto de Martino et sa réception en France", in L'homme, n. 39/151, 1999, p. 226.

61. Cfr. Giondano, Cristiana, "Translating Fanon in the Italian Context: Rethinking the Ethics of Treatment in Psychiatry", in Transcultural psycbiatry, 48, n. 3, 2011, pp. 235-239.

62. Fanon, Frantz, Peaux noires, masques blancs, Paris, Seuil, 1952, p. 68.

63. ID., Les Damnés de la terre, Paris, La Découverte, 2002, p. 240.

64. Jervis, Giovanni, "Prefazione" a Frantz Fanon, Opere Scelte, Torino, Einaudi, 1971, p. 13.

65. Cfr. Srivastava, Neelam, "Frantz Fanon in Italy: Historicizing Fanon", in Interventions, 17, n. 3, 2015, p. 310. 
aveva letto, al secondo Congresso di Scrittori e Artisti Neri di Roma, un discorso "Sulla cultura nazionale", immediatamente pubblicato su Rinascita, prima ancora di diventare un capitolo di Les damnés de la terre. Tradotto in italiano e presentato in anteprima il 15 dicembre 1961 su Paese sera, I dannati della terra veniva recensito proprio da de Martino il 10 luglio 1962.

E su quella terza pagina bisogna forse cercare le ragioni del silenzio su Fanon, pure celebrato come superamento del "marxismo classico, cioè europeocentrico"'66, e perfino come risorgimento dello

spirito rivoluzionario, languente nel vecchio continente... ormai trasmigrato nel mondo coloniale: qui infatti si manifesterebbero quella alienazione radicale, quell'estremo senso manicheo di scissione fra oppressi e oppressori, quel ricorso salutare e inevitabile alla violenza e alla eversione totali che il movimento operaio europeo è andato perdendo, cloroformizzato in parte dai compromessi della socialdemocrazia, in parte dal neocapitalismo e dai "miracoli" che lo accompagnano, in parte dal ritorno offensivo dei partiti "cristiani", e in parte infine dalle estenuanti oscillazioni fra guerra fredda e coesistenza pacifica ${ }^{67}$.

Qual è allora il problema con Fanon? La risposta è forse semplice: anche lui resta impigliato in un relativismo coloniale; troppo cauto nel separare il rivoluzionarismo algerino dal movimento operaio in Europa. De Martino sospende il giudizio:

spetterà al reale svolgimento della coscienza rivoluzionaria del mondo coloniale giudicare se l'assenza di legame col movimento operaio europeo e il molto cauto rapporto col mondo socialista [...] costituiscano una necessità o una debolezza dell'attuale processo di decolonizzazione ${ }^{68}$.

Ma forse non è neppure questo a impedire a de Martino di menzionare Fanon nella Terra del rimorso. Forse era solo il fatto che, mentre in Algeria la crisi sembrava risolversi in presa di coscienza rivoluzionaria, nell'Europa subalterna,

66. De Martino, Ernesto, "Il risveglio del terzo mondo", in Paese sera, 10 luglio 1962.

67. Ibid.

68. Ibid. Cfr anche: "It could be argued that Fanon's... idea that the European working classes were integrated into the capitalist project of domination and hence his stress on Third World peasants and urban lumpen proletariat as the only potentially revolutionary subjects, were all elements distant from the common 'structures of feeling' that emerged in European radical settings of the late 1960s" (Mellino, Miguel, "Notes From the Underground, Fanon, Africa, and the Poetics of the Real", in GIBson, Nigel (a cura di), Living Fanon: Global Perspectives, New York, Palgrave, 2011, p. 62). 
invece, un'analoga crisi rimaneva sintomo: tarantella. Era questa, d'altra parte, la differenza che sarebbe intercorsa tra "apocalissi culturali... [che] assolvono una reale funzione reintegratrice [...] [e] apocalissi psicopatologiche" ${ }^{\text {(69 }}$. Come aveva detto Fanon, citando Sékou Touré in apertura del discorso romano, "Il ne suffit pas d'écrire un chant... pour participer à la révolution" ${ }^{\text {"70 }}$.

Roberto DAINOTTO

Duke University

69. de Martino, Ernesto, La fine del mondo, cit., p. 693.

70. Fanon, Frantz, Les Damnés de la terre, cit., p. 197. 